\title{
Ethnologies
}

\section{Cultures musicales transnationales et capitalisme}

\section{Le milieu sonidero}

\section{Eduardo González Castillo}

Volume 31, numéro 1, 2009

Passages

URI : https://id.erudit.org/iderudit/038506ar

DOI : https://doi.org/10.7202/038506ar

Aller au sommaire du numéro

Éditeur(s)

Association Canadienne d'Ethnologie et de Folklore

ISSN

1481-5974 (imprimé)

1708-0401 (numérique)

Découvrir la revue

Citer cet article

Castillo, E. G. (2009). Cultures musicales transnationales et capitalisme : le milieu sonidero. Ethnologies, 31(1), 237-251. https://doi.org/10.7202/038506ar

\section{Résumé de l'article}

Selon plusieurs auteurs (Appadurai 2001 ; García Canclini 1990), l'évolution actuelle des pratiques de consommation culturelle est déterminée par des processus globaux propres à la mondialisation. À partir d'un bref survol de l'histoire de la consommation de la musique tropicale au Mexique et de l'analyse de l'une de ses manifestations les plus récentes, celle du milieu sonidero, cet article formule une critique de cette approche en accordant une attention spéciale aux dynamiques localisées et aux forces historiques et socio-économiques propres au cas qui nous intéresse. L'économie politique demeure au coeur de la présente proposition analytique.
Ce document est protégé par la loi sur le droit d'auteur. L'utilisation des services d'Érudit (y compris la reproduction) est assujettie à sa politique d'utilisation que vous pouvez consulter en ligne.

https://apropos.erudit.org/fr/usagers/politique-dutilisation/ 


\title{
Cultures musicales transnationales et CAPITALISME
}

Le milieu sonidero

\author{
Eduardo González Castillo \\ Université Laval
}

La piste est prête. Quelques camions font office de clôtures et barrent certaines rues du centre-ville de Puebla, ville coloniale mexicaine. Sortant des gros haut-parleurs, des sons graves font frémir les vitres des fenêtres des maisons environnantes. Les odeurs de colle industrielle et les lumières multicolores créent une ambiance spéciale. Amplifiée par le microphone, il y a une voix qui ne s'arrête jamais, même lors des chansons propices à la danse. Elle salue, d'un écho persistant et artificiel, les amis présents et ceux qui se trouvent loin, très loin, soit à New York, à Chicago ou encore à Los Angeles. Ce faisant, elle rend encore plus colorées les cumbias qu'on danse passionnément dans ces soirées musicales ainsi que le milieu où elles prennent place : le sonidero.

Cette note de recherche porte sur les pratiques qui structurent ce milieu apparu au Mexique dans la seconde moitié du XXc siècle et a pour but de montrer que l'analyse de ce type de pratique transnationale peut être réalisée à partir d'une perspective relevant de l'économie politique (voir Roseberry 1989 et 2002). La pertinence du cas choisi s'avère indisçutable si l'on prend en considération ses liens avec les processus sociaux reconnus par certains auteurs comme étant propres à la mondialisation. En effet, le sonidero a des liens profonds avec:

a) les dynamiques migratoires de la force de travail mexicaine, lesquelles ont donné naissance aux "communautés transnationales" (voir Kearney 1994 ; Smith 2001); 
b) la circulation des biens culturels et des technologies de la communication, qui font partie des "flux " et des réseaux de la "mondialisation" (voir Appadurai 2001 ; Cuillerai et Abélès 2002).

L'idée générale défendue ici est qu'au moment de son analyse, ce type de pratique culturelle ne doit pas être exclu du contexte socioéconomique et historique qui se trouve à son origine. Par conséquent, dans ce travail, une attention spéciale est accordée à la façon dont le milieu sonidero a été lié, tout au long de son histoire, à l'évolution récente du capitalisme au Mexique, notamment aux processus d'urbanisation, d'industrialisation et de migration de la force de travail.

Étant donné que les pratiques sonideras plaisent à certaines couches de la population mexicaine préférant la musique tropicale, l'article débute par une exploration historique de la diffusion de ce type de musique dans le pays, ainsi que par une analyse de son articulation à l'ensemble de la vie culturelle mexicaine. Par la suite, nous discuterons d'un cas spécifique, celui de l'État de Puebla, au Mexique, où le mouvement sonidero est bien répandu. Dans cette partie de l'article, l'accent est mis sur la manière dont ce loisir favorise la reproduction d'une culture populaire urbaine sur les plans de la classe sociale, du genre, des identités de quartier et des différences de génération. Enfin, quelques réflexions font office de conclusion et de synthèse.

\section{La diffusion de la musique tropicale au Mexique'}

C'est dans un Mexique postrévolutionnaire ${ }^{2}$ subissant une modernisation et une urbanisation accélérée que la consommation de la musique tropicale s'est diffusée. D'une part, l'amélioration générale des conditions de vie a permis la croissance "naturelle " de la population habitant dans les anciens centres urbains mexicains; d'autre part, encouragée par l'industrialisation du pays, la migration rurale vers les villes les plus dynamiques a favorisé un phénomène d'agglomération urbaine.

1. Les notes suivantes s'appuient sur les travaux de Moreno Rivas (et de la Condition féminine1989 ; Sevilla 1996 et Monsiváis 1980.

2. À la suite d'une décennie de guerre, la Révolution mexicaine a fini aux débuts des années 1920 pour donner lieu à l'émergence d'un État corporatiste relativement fort. 
L'urbanisation du pays a donné lieu à de nouveaux modes de vie. Elle a aussi transformé les pratiques de consommation culturelle et d'utilisation du temps libre des habitants des villes mexicaines. En reprenant l'approche proposée par Chatterton et Hollands (2002) et Hollands (2002), il est possible de penser qu'un nouveau système économique basé sur le développement de la vie nocturne a émergé. Ainsi, en quête de formes modernes d'amusement nocturne, la bourgeoisie citadine - ainsi que les classes moyennes mexicaines ont été attirées par les pratiques de consommation qui, à l'époque, caractérisaient aussi l'ambiance nocturne d'un Cuba prérévolutionnaire de même que celle des États-Unis. C'est de cette façon que des genres musicaux tels le danzón, le son cubano ou le mambo sont arrivés au Mexique.

La radio et le tourne-disque ont joué un rôle très important dans la diffusion et la généralisation du goût pour la consommation de cette musique. De même, ils ont accéléré sa propagation dans les différents espaces de la vie urbaine quotidienne et parmi les différentes couches de la société. En effet, pour des raisons financières, il n'était pas accessible à tout le monde d'aller écouter de la musique tropicale en direct dans les grands cabarets nocturnes. C'est l'utilisation collective des appareils de son électriques - que ce soit dans des occasions spéciales ou dans la vie quotidienne - qui a amené les habitants pauvres des villes à développer graduellement un intérêt pour ce genre de musique.

Pour les classes sociales aisées, la consommation de cette musique permet un rapprochement avec la vie nocturne caribéenne de la bourgeoisie cubaine et américaine. En contraste, les groupes non privilégiés semblent trouver dans la musique tropicale et dans les manières de la danser, une forme d'adaptation au mode de vie urbain. En ce sens, les pistes de danse des cabarets privés, d'un côté, et les cours intérieures des vecindades ${ }^{3}$ de l'autre, s'avèrent être les deux pôles socioéconomiques de la consommation urbaine de ce type de musique. Or, cette rigoureuse division des espaces de consommation s'est atténuée au fur et à mesure que le goût pour cette musique ainsi que la possibilité d'en écouter en direct se sont généralisés. Les grands salones de baile ${ }^{4}$ y ont joué un rôle très significatif: il s'agissait de grands espaces modestement décorés où des orchestres appelées sonoras jouaient des chansons populaires devant un public.

3. Des maisons collectives.

4. Des salons de danse. 
Les dynamiques mexicaines de consommation de la musique étrangère ont été fortement bouleversées pendant les années 1960 et 1970. À cette époque, la prospérité économique de la période précédente, soit celle de l'urbanisation, a connu un certain déclin. La principale cause de cette tendance semble se trouver dans l'échec des politiques économiques de "substitution des importations " qui avaient encouragé la croissance économique du pays dans le contexte des guerres mondiales. En outre, suite à l'avènement de la Révolution cubaine, la bourgeoisie mexicaine et l'industrie culturelle nationale se sont éloignées des productions musicales de l'île devenue socialiste. C'est ainsi que, dans le "champ " (Bourdieu 1979) de la consommation de la musique étrangère, la musique anglophone s'est substituée à la musique caribéenne chez les classes aisées mexicaines.

Dès lors, aux populaires salons de danse et aux couples aimant danser s'opposaient les petites cafétérias et l'écoute désinvolte de musique anglophone. Les mouvements rythmiques et répétitifs de la danse tropicale n'attiraient plus les corps extatiques et convulsés des passionnés de rock. Par ailleurs, bien que des processus de circulation culturelle entre classes sociales aient aussi eu lieu dans le cas de la musique anglophone, l'impossibilité de comprendre les paroles des chansons représentait — du moins à l'époque - une barrière empêchant la diffusion de ce genre de musique chez les classes populaires.

Bonfil Batalla (1990) parlait de la négation de l'indigène au sein du projet de construction du Mexique postrévolutionnaire. Selon cette perspective, il devient pertinent de se demander si cet abandon de la consommation de la musique tropicale ne faisait pas partie d'une stratégie de négation raciste des pratiques culturelles de la population urbaine d'origine indigène et/ou rurale. De même, la diffusion du discours développementaliste durant le deuxième après-guerre (voir Escobar, 1995), semble aussi avoir renforcé l'imaginaire mexicain par rapport à la distinction ségrégative entre la musique des "sousdéveloppés" (à éviter) et celle des pays développés (convoitée).

La cumbia, d'origine colombienne, et la salsa, issue de Porto Rico, sont apparues au Mexique dans les années 1970-1980, au moment où le marché symbolique de la musique étrangère avait déjà été transformé. La nouvelle hiérarchie ne leur laissait aucune chance d'atteindre, chez les élites du pays, les places autrefois occupées par le mambo ou le danzón. Par ailleurs, la détérioration des conditions de vie de la 
population urbaine et rurale mexicaine ne semblait pas favoriser une revalorisation nostalgique à cet égard. Les pauvres étaient de plus en plus miséreux et leurs pratiques ainsi que leurs manières d'utiliser l'espace urbain devenaient indésirables pour les classes hautes et moyennes.

Dans les années 1980, la pauvreté, la pollution et le manque de services se généralisent et marquent la vie urbaine mexicaine. La baisse du prix international du pétrole (1982), la dévaluation du peso, l'inflation et l'augmentation du chômage frappent durement les habitants les plus défavorisés du pays. Le gouvernement mexicain tente de remédier à cette situation en délaissant les politiques sociales au profit des forces du capitalisme mondial en favorisant l'intégration de l'économie nationale au marché international. En vertu de cela, les capitaux privés nationaux et internationaux ont commencé à acheter diverses entreprises étatiques, et le marché des biens de consommation s'est davantage ouvert aux produits étrangers.

L'adaptation des classes défavorisées à ces conditions de vie difficiles a donné lieu à la croissance d'activités commerciales (formelles et informelles) et à d'autres stratégies de survie. La vente d'articles électroniques entrés illégalement dans le pays (contrebande) a été l'une de ces stratégies, mais c'est sans doute la migration internationale qui constitue la réaction populaire la plus importante face aux conditions de l'économie nationale. En témoigne l'augmentation du nombre de Mexicains qui, pendant la décennie des années 1980, quittent le pays pour migrer illégalement aux États-Unis. Il est aussi important de noter la participation croissante aux flux migratoires vers le Nord des Mexicains habitant dans les villes (voir CONAPO 2006).

L'accessibilité grandissante aux biens électroniques, l'émergence de nouvelles activités économiques de survie et, enfin, l'augmentation de la migration mexicaine illégale vers les États-Unis ont favorisé, dans le domaine de la consommation culturelle, une sorte de transformation technologique des pratiques de loisir chez les classes populaires. D'ailleurs, à l'époque, la cumbia était déjà devenue la musique la plus appréciée des classes populaires au travers le pays. En effet, par opposition à la salsa - dont la performance exige beaucoup de musiciens et une grande diversité d'instruments - la simplicité instrumentale et musicale de la cumbia permet de pouvoir l'interpréter sans beaucoup d'expérience en tant que musicien ou sans grands moyens financiers pour acheter des instruments. Aux yeux des classes aisées, cette musique s'avérait déjà - comme aujourd'hui - le genre musical le plus discrédité 
dans le pays. Pour ces groupes, les vedettes locales et nationales de la cumbia et leurs adeptes représentaient le stéréotype du "naco» mexicain, c'est-à-dire du citadin pauvre d'origine indigène (Bonfil 1990).

Toutefois, dans un contexte de pauvreté extrême, la créativité des classes populaires ne s'est pas arrêtée là. C'est en fait le phénomène migratoire transnational qui a donné lieu à une autre modification des pratiques de consommation de cette musique à la fin des années 1980 . Nous faisons ici référence à l'émergence ou à la prolifération massive de sonidos (littéralement, "des sons »). Il s'agit en réalité d'entreprises familiales qui possèdent des appareils de son et de lumière et qui offrent leurs services en échange d'argent (González 2003). Son origine principale est sans doute l'usage collectif d'appareils domestiques audio dans l'espace urbain (par exemple dans les patios des vecindades). Au fil du temps, les sonidos ont rendu possible l'apparition de divers services qui répondaient à leurs exigences économiques: publicité, logistique, entretien des appareils, etc. C'est l'ensemble de ces services et activités que l'on appelle communément le "milieu sonidero": une ambiance de consommation musicale véritablement distincte des formes précédentes, car il s'agit d'un des premiers espaces transnationaux de consommation musicale créé par les habitants pauvres du Mexique et ceux émigrés aux États-Unis.

Ainsi, divers centres urbains mexicains et américains (tels ceux de New York, Chicago ou Los Angeles) ont connu l'émergence de nouvelles manières de consommer, de vendre et de produire les biens musicaux très précieux pour certains Mexicains vivant dans la pauvreté. À cet égard, les migrants ont contribué et participé à la diffusion du goût pour cette musique par des transferts d'argent et l'achat de matériel audio. Les habitants des villes mexicaines ont pour la plupart développé un ensemble de pratiques commerciales et culturelles donnant lieu à une sorte de microindustrie culturelle (voir González 2003) " alternative », mais ni "indépendante", ni «contestataire " (voir Williams 1997).

À l'heure actuelle, les danses sonideras peuvent être réalisées dans des espaces très diversifiés, car elles sont itinérantes et font partie des pratiques d'appropriation des espaces publics (comme la rue ou les esplanades) de la part des classes populaires urbaines. Cela a donné lieu à des tensions entre les adeptes du milieu et les divers groupes d'habitants de la ville qui associent généralement les sonideros à la 
délinquance et à la consommation de drogues. À cet égard, le milieu sonidero est tout aussi "dénigré " que la cumbia dans le champ de la consommation musicale au Mexique.

Sa distance par rapport aux industries culturelles dominantes; son autonomie face aux politiques culturelles nationales; ses rapports étroits avec les pratiques économiques populaires de survie, et le caractère clandestin de sa production et de sa consommation (piraterie, appropriation illégale des lieux publics et altération de ses usages " adéquats") ont valu au milieu sonidero d'être considéré comme l'un des espaces les plus décriés de la consommation musicale au Mexique.

\section{Les sonidos à Pueb/a ${ }^{5}$}

Puebla est une ville mexicaine située au centre du pays et qui compte à l'heure actuelle environ deux millions d'habitants. Deux facteurs socio-économiques ont rendu possible l'apparition des sonidos dans cette ville. Premièrement, la montée, dans les années 1980, de l'économie populaire urbaine qui s'est traduite par la prolifération d'espaces de commerce visant les classes défavorisées locales: des zones de commerce ambulant ou des centres commerciaux populaires. C'est dans ces types d'espaces commerciaux que les principaux représentants du milieu ont proliféré : les personnes en charge de la "publicité ", les ateliers d'électronique, les techniciens et les propriétaires des équipements électroniques. Deuxièmement, il y a eu le soutien financier des émigrants de la ville qui, face aux difficiles conditions de vie du pays, ont fait augmenter la migration urbaine illégale vers les ÉtatsUnis. Les migrants de Puebla se trouvent surtout à New York, où les sonidos mexicains réalisent aussi des prestations. La plupart des sonidos de Puebla s'avèrent être de petites entreprises économiques, pullulant partout dans les zones pauvres de la ville et constituées de six ou sept personnes: le propriétaire, le locuteur et le reste des travailleurs (qui effectuent le montage de l'équipement électronique).

Comme mentionné ci-haut, les sonideros développent leurs activités dans un contexte de relation conflictuelle avec les sphères de pouvoirs de la ville. D'une part, étant donné la légalité douteuse de leurs marchandises (la musique enregistrée), les sonidos sont considérés comme des "pirates " musicaux, situation qui entraîne la surveillance et parfois même l'interdiction de leurs activités. D'autre part, les danses

5. Pour savoir plus au sujet de ce milieu culturel, voir González 2003. 
sonideras, réalisées souvent sans l'autorisation de la municipalité, sont fréquemment associées à la criminalité et à la violence. Néanmoins, face à des politiques publiques hostiles, les sonideros ont constitué un espace de loisir qui, comparativement aux habitudes de consommation des autres groupes et classes sociales à Puebla, caractérise la ville populaire et alternative.

En ce sens, malgré l'existence de plusieurs lieux fermés où les danses sonideras pourraient être réalisées, ce sont les espaces ouverts des quartiers populaires - la rue ou les esplanades publiques - qui sont choisis par les sonideros pour leurs soirées. En général, on barre les rues pour délimiter la piste de danse en se servant de différents types d'obstacles (des camions par exemple). Les jours de danse, les sonidos qui animeront la soirée se rencontrent le matin pour assembler leur matériel. Le soir venu, quand les participant(e)s se réunissent, il est déjà possible d'entendre les tests de son des sonidos. Les gros haut-parleurs commencent à faire vibrer les zones adjacentes de la ville et le rythme sonidero résonne dans l'ensemble de la ville.

Les adeptes des sonidos arrivent graduellement à la tombée de la nuit. Avant le début de la danse, les personnes bavardent et se tiennent aux abords de la "piste " et lorsque la voix du sonido annonce le commencement de l'enregistrement sonore de la danse, les participant(e)s s'agglutinent sur la piste en trémoussant leurs corps avides de danse. Ceux et celles qui ne dansent pas boivent et s'amusent en plaisantant à propos de tout et de rien. Quelques-uns inhalent, dans leur coin, de la colle industrielle.... Personne ne dit rien, on tolère les vices d'autrui. Les danses prennent fin au matin et les participant(e)s rentrent chez eux en commentant les anecdotes de la soirée.

À Puebla, la manière de danser des sonideros est une variante de ce qu'on pourrait appeler le style "classique » mexicain de la danse de la cumbia. En général, ce style consiste en la formation de couples qui, liés par les bras, bougent leur corps, tournent et effectuent de petits sauts au rythme de la musique. L'un des deux dirige la danse. Les sonideros ont accentué ces aspects de la danse en faisant des pas ou des sauts plus grands ou en inventant divers types de tours. Leur innovation la plus visuellement spectaculaire est sans doute la danse à trois personnes. Elle reproduit le modèle "classique " qui implique qu'un membre du couple (généralement l'homme) dirige l'(es) autre(s). Cette façon de danser permet au corps d'exprimer et de reproduire différentes choses: des habitus qui, dans les termes de Bourdieu (1979), expriment, en se 
reproduisant, les positions occupées par les groupes sociaux dans l'espace social en général. Intéressons-nous à quatre aspects de la condition sociale reproduite lors de danses sonideras : ceux de la classe sociale, des rapports de genre, des identités de quartier et des identités générationnelles. Ces dimensions s'insèrent dans une approche liée à l'économie politique. En effet, elles sont fortement liées à l'ensemble du contexte socio-économique qui régule les rapports de pouvoir et les inégalités à l'intérieur de l'espace urbain.

\section{La classe sociale}

Une des dimensions les plus englobantes de l'expérience des conditionnements sociaux est celle de la classe sociale, celle de la position que les individus occupent dans la société en fonction de la structure et du volume de leurs capitaux économiques et symboliques (Bourdieu 1979). En ce qui concerne les capitaux dominants (richesse économique, scolarité), les personnes qui fréquentent le milieu sonidero poblano s'avèrent marginales. Malgré leur jeune âge - la plupart d'entre eux ne dépassent pas la vingtaine - les sonideros cumulent de longues expériences de travail et de courts trajets scolaires. Il s'agit d'individus qui, quand ils ont la chance d'avoir un emploi, travaillent comme livreurs dans les marchés populaires, comme employés dans de petits ateliers (dans le cas des garçons) ou comme commis d'épicerie (dans le cas des filles).

La caractéristique distinctive de la danse des sonideros est la position courbée du corps et le caractère lâche mais rapide, des mouvements effectés. Il s'agit d'une allure dansante tout à fait divergente de celle promue par les classes plus aisées, qui ne cessent d'imiter. les modèles corporels diffusés par l'industrie culturelle nord-américaine. La discrimination qu'ils subissent a amené les adeptes du milieu à développer une forte conscience du caractère marginal de leur loisir. Ainsi, quand les sonideros parlent du type de personnes qui assistent aux danses sonideras, ils font allusion à l'absence de fresas [fraises], c'est-à-dire de jeunes petits bourgeois mexicains.

Pendant les danses, l'homogénéité de classe se manifeste dans l'ambiance créée. Pour les sonideros, il est naturel ou normal de fermer illégalement la rue pour danser ("pourquoi pas ? »), ou de danser dans un entourage saturé par l'odeur de colle. Toute question à ce propos peut être évacuée comme non pertinente. En ce sens, le corps sonidero dansant est héritier d'une tradition acquise par les habitants pauvres de 
la ville depuis leur intégration dans l'espace urbain. L'identification corporelle qui se réalise pendant la danse de la cumbia, soit le fait de pouvoir harmoniser les mouvements des différents corps, reproduit une tradition corporelle "prolétaire ". Une pratique d'amusement adoptée depuis longtemps par les émigrant(e)s ruraux qui ont peuplé cet espace urbain.

\section{Les rapports entre les sexes}

La position subordonnée que les femmes occupent dans la société mexicaine est nuancée par les particularités culturelles du milieu sonidero. Ici, comme Bourdieu l'a souligné dans La domination masculine (2002), la logique du marché des biens symboliques continue à donner aux femmes le rôle "d'objets " - et non d'agents — dans l'échange symbolique. Par exemple, dans les produits élaborés par les publicistes sonideros, l'image de la nudité féminine est utilisée comme un "appât " dont la fonction principale est celle d'inciter les hommes à consommer les biens du milieu.

Dans ce dernier, en général, la participation des femmes est éclipsée par celle des hommes. Bien que les sonidos soient dans la plupart des cas des entreprises familiales, ils sont toujours présentés comme des entreprises masculines. De telle sorte que, habituellement, le nom du "chef " de famille est utilisé pour nommer le sonidos (Ex. "Sonido Fantasma de Alejandro Juárez ", "Sonido Reebock de Armando Cuautle »). Malgré cela, pendant les danses, la communication des corps masculins et féminins entrâne une identification créatrice et solidaire. Créatrice parce que l'entrecroisement des individus donne lieu à une innovation constante dans le port du corps et, par conséquent, d'expérimenter la coexistence corporelle entre les sexes.

Les pas et les mouvements des corps favorisent d'autre part la solidarité entre les genres en tant que membres d'une même classe sociale, c'est-à-dire, en tant que produits de différentes expériences de genre, mais réunis en raison de leurs origines sociales et de leurs situations dans l'espace social. En dansant, les corps explorent les différentes possibilités expressives de leurs charges sociales et arrivent à un état d'amusement qui n'implique pas nécessairement l'effacement des différences de genre. En effet, c'est l'homme qui dirige la danse et c'est lui qui se démarque régulièrement aux dépens de sa partenaire devenue un accessoire de la grâce masculine. Celui qui maîtrise véritablement la danse sera celui qui saura s'y prendre avec deux filles en même temps. 
Pour l'homme donc, l'exécution de la danse implique aussi le risque potentiel de perdre son prestige s'il n'arrive pas à bien diriger la danse.

\section{Identités de quartier}

Malgré les affirmations d'auteurs comme Arjun Appadurai (2001) à propos de la déterritorialisation des pratiques culturelles dans le contexte de la transnationalité, le milieu sonidero - un milieu, comme nous l'avons déjà vu, influencé par les mouvements de migration transnationale - n'a pas déterritorialisé les identités liées à l'espace public qui lui sont associées. Ainsi, si dans les danses les identités des adeptes sont exprimées en fonction de la collectivité à laquelle ils font partie, cette collectivité est toujours en rapport étroit avec le quartier d'origine. L'appartenance aux arrondissements populaires est mise en valeur par les saludos (salutations) que, à la demande des assistant(e)s, le locuteur envoie continuellement par le microphone (par exemple "Salutations aux salseros de la 8 poniente ", "Salutations aux guerriers de la Guadalupe Hidalgo ») et qui sont enregistrées sur des cassettes que l'on pourra trouver les jours suivants dans les marchés populaires.

L'enregistrement sonore de la soirée permet une inscription plus durable de la présente dans l'espace urbain et de son utilisation. La courte durée de la soirée est immortalisée dans la mémoire technologique et la banda [bande], en se procurant ces enregistrements, pourra se remémorer sa participation à la danse. Ainsi, cette dernière ne s'évapore pas avec la fin de la soirée, mais est conservée en mémoire par les habitant(e)s de la ville.

L'appartenance aux arrondissements populaires peut engendrer une extrême camaraderie entre les divers groupes. La circulation des boissons, par exemple, représente l'expression la plus sensible d'amabilité entre les participant(e)s - se connaissant ou étant étrangers. De telle sorte qu'un même pot de bière, par exemple, peut circuler entre différents groupes en signe d'amitié. Ainsi, les lèvres des participant(e)s touchent les mêmes verres et bouteilles, et créent des alliances par des liens de salive. Le corps est donc le principal véhicule de cette affectivité. Les accolades, les salutations et les baisers en sont les principales manifestations. Par contre, la camaraderie disparaît instantanément lorsqu'il y a des problèmes entre deux inconnus et les groupes respectifs auxquels appartiennent les individus interviennent brusquement pour soutenir (hacer el paro) leurs amis impliqués. 


\section{Identité générationnelle}

D'après les sonideros, la manière dont ils dansent se distingue fortement de celle des générations antérieures. C'est le caractère plus accéléré de leurs mouvements qui, à leur avis, rend la danse sonidera très différente de la façon dont leurs parents dansaient sur la musique des anciens groupes qui jouaient les cumbias. Pour les sonideros, les groupes musicaux, les salons de danse et la cumbia la plus lente font partie du passé. Il y a donc, dans l'expérience sonidera, un sens d'actualité, de mise à jour des pratiques d'amusement héritées des premières générations de la société urbaine "massifiée " (voir Monsiváis 1980 et Romero 1976). En ce sens, les sonideros mettent en valeur le caractère plus rythmé de leurs pas de danse comme la différence la plus significative entre leur façon de danser et celle de leurs parents.

Cependant, le lien culturel entre les générations est indubitable et, malgré toutes les innovations introduites par les sonideros dans le domaine de la consommation musicale, leur pratique d'amusement reproduit et actualise les positions sociales occupées par les anciens passionnés de la cumbia. Il s'agit, comme nous l'avons déjà mentionné, d'une situation sociale plutôt marginale. Dans ce même ordre d'idées, il est possible de dire que le corps sonidero reproduit les dispositions culturelles héritées d'une culture urbaine/ouvrière - la manière de danser, les perceptions à l'égard de la position de la femme par rapport à l'homme dans la danse (et dans la vie sociale) - - et les adapte au contexte social actuel, dont les caractéristiques sont : la migration, le chômage et l'accessibilité aux nouvelles technologies.

\section{Conclusion}

La présente analyse soulève des questions importantes concernant l'étude des pratiques culturelles transnationales. Quelle est la relation, par exemple, que ce type de pratique entretient avec l'évolution récente du capitalisme ? Quels sont les rapports de ces milieux culturels en mobilité constante avec les différents contextes régionaux ?

L'analyse historique de la consommation de la musique tropicale au Mexique nous a permis de mieux saisir les liens qu'entretiennent les sonideros avec l'évolution socio-économique et culturelle de la société mexicaine. Cela nous a permis de montrer que l'importance actuelle du milieu sonidero réside dans sa liaison avec divers changements au sein des pratiques de consommation de la musique dans ce pays. En ce 
sens, on a discuté de la formation d'une culture musicale urbaine, reproduite grâce à diverses conditions locales, régionales, nationales et transnationales. Dans le cas de Puebla, ces conditions ont été liées à l'expansion de l'économie populaire urbaine (une économie de survivance), à l'augmentation de la migration internationale et à la présence d'une culture urbaine marginale, active sur différents plans de la vie sociale (classe, relations entre les sexes, identité de quartier et le facteur générationnel).

En outre, notre analyse a aussi montré la pertinence et la nécessité d'une approche "matérialiste » en ce qui a trait à l'étude des cultures transnationales contemporaines. L'évolution économique du Mexique et, de manière encore plus spécifique, les changements sur le plan des déplacements de la force de travail, témoignent de l'importance du capitalisme dans la formation et de la transformation des pratiques culturelles contemporaines. De cette façon, la migration rurale/urbaine de la première moitié du $\mathrm{XX}^{\mathrm{e}}$ siècle (diffusion du goût pour la musique tropicale) et la migration transnationale actuelle (formation du milieu sonidero), s'avèrent des sources d'innovation pour les pratiques de consommation musicale des habitant(e)s du Mexique. Ces deux grands déplacements historiques de la force de travail mexicaine, changements majeurs en ce qui concerne l'insertion dans le marché du travail dans le capitalisme mexicain et global, ont donné lieu à des conditions sociales, économiques et technologiques favorables à la création d'une culture musicale propre. C'est, sans doute, l'économie politique qui peut le mieux nous permettre de comprendre et d'expliquer les particularités culturelles, historiques et socio-économiques d'un tel processus. 


\section{Références}

Appadurai, A., 2001, Après le colonialisme. Les conséquences culturelles de la globalisation. Paris, Payot.

Bonfil, G., 1990, México profundo. Una civilización negada. México, Grijalbo-Consejo Nacional para la Cultura y las Artes.

Boudieu, P., 1979, La distinction. La critique sociale du jugement. Paris, Éditions Minuit.

, 2002, La domination masculine. Paris, Seuil.

Chatterton, P. et R. Hollands, 2002, "Theorising Urban Playscapes: Producing, Regulating and Consuming Youthful Nightlife City Spaces ". Urban Studies 39 (1) : 95-116.

CONAPO, 2006, Migración mexicana hacia Estados Unidos. http:// www.conapo.gob.mx/mig_int/03.htm, page consultée le 17 octobre 2006.

Cuillerai, M. et M. Abélès, 2002, «Mondialisation. Du géo-culturel au biopolitique ". Anthropologie et Sociétés 26 (1) : 11-28.

Escobar, A., 1995, Encountering Developement. The Making and Unmaking of the Thrid World. Princeton, Princeton University Press. Garcia Canclini, Néstor, 1990, Culturas híbridas. Estrategias para entrar y salir de la modernidad. México, GRIJALBO.

Gonzàlez, E., 2003, "Sonidero poblano ». Mirada antropológica 1: 96 116.

Hollands, R., 2002, "Divisions in the Dark. Youth Cultures, Transitions and Segmented Consumption Spaces in the Night-Time Economy ». Journal of Youth Studies 5 (2) : 153-171.

Kearney, M., 1994, "Transnationalism. From Hypen-nation and Profannation to Transnation ", document du symposium: "Transnationalism, Nation-State Building and Culture». Motel Mijas, Mijas, Espagne.

Monsivais, C., 1980, «Los de Atrás se Quedarán (Notas sobre Cultura y Sociedad de Masas en los Setentas)». Nexos 26 (35):4.

Moreno, Y., 1989, Historia de la música popular mexicana. México, CONACULTA.

Romero ,José Luis, 1976, Latinoamérica. Las ciudades y las ideas. México, Siglo XXI.

Roseberry, W., 1989, Anthropologies and Histories. New Brunswick, Rutgers. 
—_ 2002, "Understanding Capitalism - Historically, Structurally, Spatially ». Dans D. Nugent (dir.), Locating Capitalism in Time and Space. Global Restructurings, Politics, and Identity : 61-79. Stanford, Stanford University Press.

Sevilla, A., 1996, "Aquí se siente uno como en su casa. Los salones de baile popular de la ciudad de México ». Alteridades 6 (11) : 33-41. Smith, M., 2001, Transnational Urbanism. Locating Globalization. New York, Blackwell.

Williams, R., 1997, Marxismo y literatura. Barcelona, Peninsula. 